\title{
Disparités en matière de santé en cancérologie: exploration de perspectives canadiennes, américaines et internationales
}

Selon sa vision, l'ACIO/CANO «joue un rôle de premier plan au niveau national et exerce une influence internationale quant à la promotion de l'excellence des soins infirmiers dans tous les domaines de la lutte contre le cancer». Toujours selon elle, «les Canadiens, peu importe où ils en sont dans le continuum du cancer, accèdent en temps opportun à des soins infirmiers de haute qualité» (ACIO/CANO, p. 8*). C'est dans cette optique qu'un groupe d'experts représentant toute une gamme de perspectives nationales et internationales a été formé en vue d'explorer les iniquités en matière de santé dans le domaine de la cancérologie lors de la 25 e conférence annuelle de l'ACIO/CANO tenue à Vancouver en octobre 2013. Le groupe se composait des experts suivants en recherche sur les disparités en matière de santé (Colleen Varcoe et Christina Sinding), anciennes présidentes de l'Oncology Nursing Society (ONS) (Mary Gullatte) et de l'International Society of Nurses in Cancer Care (ISNCC) (Greta Cummings) et, une experte du Partenariat canadien contre le cancer (Margaret Fitch). Les infirmières et infirmiers en oncologie sont exceptionnellement bien placés pour exercer une influence positive sur léquité dans les soins de santé en cancérologie et ce, aux niveaux national et international. Les présentations des membres du groupe sont résumées ci-dessous.

\section{Disparités en matière de santé en cancérologie: concepts fondamentaux}

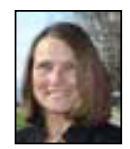

par Colleen Varcoe, inf., Ph.D. (avec Sanzida Habib, Ph.D.)

$I^{\prime}$

1 est passionnant de voir que les infirmiers et infirmières Len oncologie portent une attention accrue à l'équité dans les soins de santé et à la justice sociale. De telles préoccupations n'ont dominé ni la recherche ni la pratique en lien avec le cancer; il se peut que cela tienne, en partie, au fait que le cancer véhicule un stigmate social moindre que des enjeux de santé où le manque d'équité est au premier plan tels que les problèmes de santé mentale, la violence ou le VIH (avec des exceptions comme le cancer du poumon chez les fumeurs). Les infirmières et infirmiers en oncologie portent une attention accrue aux iniquités (ou disparités) en matière de santé à une époque où la compréhension des concepts fondamentaux se consolide. Le but de la présente introduction est de passer en revue les concepts fondamentaux de l'intégration des

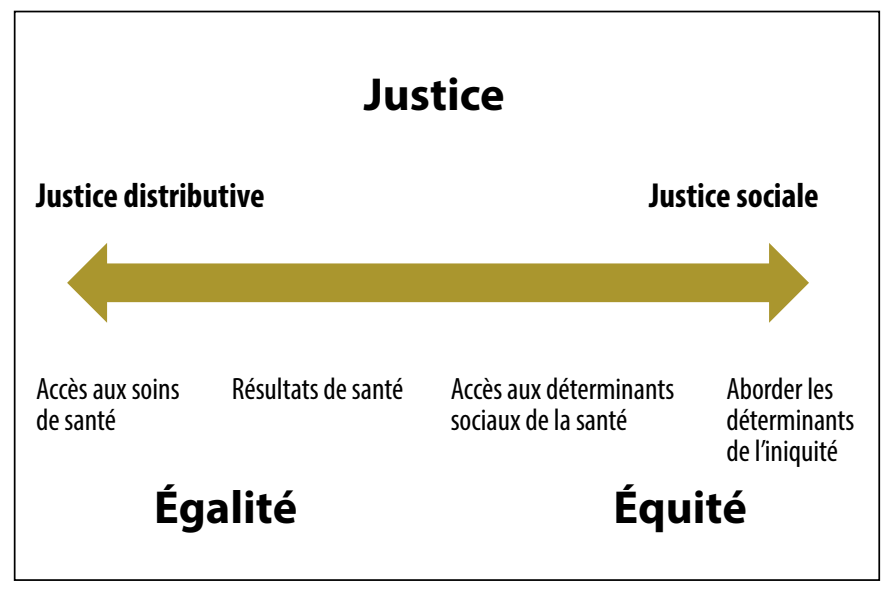

Figure I: Équité, égalité et justice dans les soins de santé iniquités en matière de santé dans la recherche et la pratique en soins de santé et d'examiner leur pertinence relativement à la cancérologie.

Les termes disparité et iniquité sont souvent utilisés de façon interchangeable, mais ils doivent tous deux être distingués du terme inégalité. Inégalité est un terme générique qui fait référence aux différences entre des groupes, que celles-ci soient justes ou non. Les iniquités ou disparités font référence aux différences qui: a) sont injustes et donc une préoccupation morale, b) sont causées par des arrangements sociaux, et c) peuvent être éventuellement rectifiées (Whitehead \& Dahlgren, 2006).

Cet entendement des iniquités se base sur une conception critique de la justice sociale, laquelle diffère de l'idée de justice distributive qui étaye une bonne partie de la pensée occidentale liée à léthique, à la recherche et à la pratique en soins de santé. La justice distributive s'intéresse à la répartition équitable des ressources dans la société (Hoedemaekkers \& Dekkers, 2003). La justice distributive examine en premier lieu les ressources disponibles au sein d'une société et évalue la répartition dont elles font l'objet parmi les groupes. Il est important de noter qu'en ce qui concerne les soins de santé, la justice distributive attire l'attention sur l'équité de l'accès aux soins de santé. Par contre, la justice sociale se préoccupe d'abord de comprendre les différences entre sous-groupes, notamment sur le plan du pouvoir, et souligne les conditions structurales qui façonnent les iniquités (Young, I990, 200I). Par exemple, l'examen de la prévention du cancer du sein ou du cancer du col utérin dans l'optique de la justice distributive braque les projecteurs sur le nombre de praticiens et d'installations de dépistage, de traitement et de services de laboratoire et sur leur répartition. Le regard porté par la justice sociale examine les positions différentes de groupes et/ou sous-groupes particuliers et la manière dont celles-ci façonnent l'accès aux soins de santé et les résultats de santé. 
La justice sociale, quant à elle, commence par le social plutôt que par les biens à repartir. Comme Kirkham et Browne (2006) l'expliquent: «...alors que l'aspect justice s'intéresse à ce qui est juste ou non, l'aspect social attire l'attention sur l'application du concept de justice aux groupes sociaux, [et] fait apparaître au grand jour la manière dont la justice et les injustices sont entretenues par les institutions et les relations sociales; et souligne la mesure dans laquelle l'expérience individuelle s'imbrique dans le plus vaste domaine que sont les complexités politiques, économiques, culturelles et sociales» (p. 325, traduction libre). L'utilisation d'une optique de justice sociale élargit la compréhension de l'accès aux soins de santé et, comme la figure i le suggère, amène les observateurs à dépasser la simple équité en matière d'accès aux soins pour examiner également les résultats de santé et l'accès aux déterminants sociaux de la santé, d'une part, et des iniquités, d'autre part. En cancérologie, une optique de justice sociale concentre l'attention sur le fait que le risque de cancer et les résultats afférents sont façonnés par les déterminants sociaux de la santé et les iniquités. Par exemple, dans une optique de justice sociale, on conçoit que les différences sur le plan des habitudes tabagiques entre sous-groupes sont façonnées par les rapports de forces liés à l'âge, au sexe, à l'emploi, aux capacités, au racisme, aux relations coloniales et à la pauvreté.

Pour être menées dans une optique de justice sociale, la recherche et la pratique exigent que l'attention porte sur les déterminants sociaux de la santé et les iniquités. En étudiant le problème $\mathrm{du}$ moindre accès au dépistage du cancer du sein et du cancer du col utérin chez les femmes sud-asiatiques, Habib (20II) ne s'est pas contentée d'examiner les connaissances des femmes et les barrières linguistiques qui sont habituellement utilisées pour expliquer le moins bon accès aux services de prévention et les plus mauvais résultats de santé. Elle a découvert que l'accès au dépistage du cancer et à d'autres services de santé était façonné par une combinaison de dynamiques notamment les normes et les rôles fondés sur le genre, la catégorie d'admission des immigrants au Canada, l'âge, la stabilité/l'instabilité financière, le niveau de scolarité et de compétence linguistique en anglais, l'accès à un revenu, à des services d>établissement et à d'autres ressources. Elle a attiré l'attention sur la manière dont des arrangements sociaux injustes liés à la race, à la classe et au statut d'immigrant(e) s'entrecroisent avec le genre de façons bien diverses et complexes dans la vie matérielle et quotidienne des femmes sud-asiatiques pour les placer dans des situations radicalisées

\section{RÉFÉRENCES}

Farmer, P. (2003). Pathologies of power: Health, human rights, and the new war on the poor. Berkeley: University of California Press.

Habib, S. (20II). South Asian immigrant women's access to and experiences with breast and cervical cancer screening services in Canada. (Doctoral dissertation), University of British Columbia, Vancouver.

Hankivsky, O., \& Christoffersen, A. (2008). Intersectionality and the determinants of health: A Canadian perspective. Critical Public Health, 18(3), 27I-283. et défavorisées, dans la catégorie des «autres», où l'accès au dépistage du cancer à titre préventif devient un défi de taille.

Les concepts de justice sociale et d'iniquités/disparités en matière de santé sont dûment complétés par deux autres concepts: l'intersectionnalité et la violence structurale. L'intersectionnalité propose une critique de la primauté de n'importe quelle catégorie sociale (comme la race, la classe, le genre, l'aptitude, la masse corporelle, la géographie) et concentre l'attention sur le caractère inséparable des rapports de forces entre ces dimensions (Hankivsky \& Christoffersen, 2008; Varcoe, Pauly, Laliberte, \& MacPherson, 20II). L'intersectionnalité conçoit ces catégories et systèmes comme étant simultanément co-construits aux niveaux macro (institutions sociales) et micro (identités individuelles) (Weber \& Parra-Medina, 2003). Il met explicitement l'accent sur les différences entre les groupes et en leur sein et cherche à mettre au jour les facteurs sociaux dont l'interaction touche la vie des êtres, notamment la position sociale, l'état de santé et la qualité de vie (Hankivsky et al., 20IO). La violence structurale se réfère aux arrangements sociaux nocifs qui servent les intérêts des puissants et sont profondément ancrés dans les organisations politiques et économiques tels que la pauvreté extrême et relative, le racisme et la discrimination fondée sur le sexe qui se traduisent par de nombreux cas de violation des droits de la personne (Farmer, 2003). Ensemble, ces idées font que les analyses délaissent désormais l'appartenance à des groupes pour privilégier les conditions de marginalisation (passant ainsi, par exemple de «race» à «racisme»). Pour donner un exemple, plutôt que de chercher à expliquer les écarts au niveau des résultats liés aux soins anticancéreux chez les femmes sud-asiatiques, on s'efforce de cerner des explications au niveau des effets des arrangements sociaux et organisationnels associés au sexe, à la migration, au racisme, etc.

Dans une optique de justice sociale, les solutions aux problèmes de santé se situent «en amont». Cela signifie que lorsque les intersections complexes parmi les arrangements sociaux sont perçues comme étant la cause profonde de problèmes de santé, il est nécessaire d'aborder ces arrangements. $\mathrm{Au}$ lieu de supposer que le manque de compréhension ou les barrières linguistiques permettent d'expliquer les différences dans l'accès au dépistage du cancer et au lieu donc de produire dans diverses langues davantage de brochures sur le dépistage du cancer, une optique de justice sociale oriente les fournisseurs de soins à aborder des facteurs structuraux comme le racisme systémique dans le monde des soins de santé.

Hankivsky, O., Reid, C., Cormier, R., Varcoe, C., Clark, N., Benoit, C., $\&$ Brotman, S. (2010). Exploring the promises of intersectionality for advancing women's health research. International Journal for Equity in Health, 9, I-I5. doi:Io.II86/I475-9276-9-5

Hoedemaekkers, R., \& Dekkers, W. (2003). Justice and solidarity in priority setting in health care. Health Care Analysis, 11(4), 325-343.

Kirkham, S.R., \& Browne, A.J. (2006). Toward a critical theoretical interpretation of social justice discourses in nursing. Advances in Nursing Science, 29(4), 324-339. 
Varcoe, C., Pauly, B., Laliberte, S., \& MacPherson, G. (20II). Intersectionality, social justice and policy. In O. Hankivsky (Ed.), Intersectionality-type Health Research in Canada (pp. 33I-348). Vancouver: UBC Press.

Weber, L., \& Parra-Medina, D. (2003). Intersectionality and women's health: Charting a path to eliminating disparities. Advances in Gender Research, 7, I8I-230.

Whitehead, M., \& Dahlgren, G. (2006). Levelling up (part I): A discussion paper on concepts and principles for tackling social

\section{Disparités en cancérologie} par Christina Sinding, Ph.D.

$\mathrm{C}$ ertains chercheurs affirment que la meilleure façon de régler les disparités associées au cancer consiste à régler les disparités sociales - c.-à-d. aborder les facteurs structuraux «en amont» tels que décrits par Colleen Varcoe (ailleurs dans ce numéro). De nombreuses études montrent que le revenu, la scolarité, l'âge, le sexe, l'appartenance ethnique et le lieu de résidence sont liés à la survie au cancer. Le stade de la maladie importe au sein de cette association-en effet, les individus qui commencent à recevoir des soins quand leur cancer est plus avancé n'obtiennent pas, de manière générale, d'aussi bons résultats de santé et les individus qui sont défavorisés du point de vue social voient leur cancer diagnostiqué plus tard que leurs pairs plus favorisés.

Certains chercheurs avancent donc que le meilleur moyen de régler les disparités liées au cancer est de régler les disparités sociales. Les initiatives de promotion de l'équité telles que les campagnes en faveur d'un salaire suffisant se déroulent dans de nombreuses villes du pays et méritent notre soutien de même que les efforts visant à améliorer les paiements de transfert qui atténuent l'inégalité entre pauvres et riches, entre hommes et femmes et entre blancs et individus racialisés (Stanford, 2008). Les liens entre certains emplois et le cancer sont également pertinents sur le plan des disparités face au cancer. Des recherches effectuées dans la région de Windsor ont révélé, par exemple, un risque élevé de cancer du sein chez les femmes travaillant dans l'agriculture, la fabrication de plastique pour véhicules automobiles et les conserveries alimentaires (Brophy et al., 20I2). Les infirmières et infirmiers en oncologie qui s'intéressent à ces enjeux pourront regarder un documentaire convaincant de Sandra Steingraber, une écologiste et une survivante du cancer, intitulé Living Downstream (Steingraber, 20I4).

Tandis que nous faisons entendre nos voix concernant ces enjeux plus vastes, nous devons également œuvrer envers la transformation du système de soins de santé puisque les disparités face au cancer ne surviennent pas seulement lorsque le cancer de l'un est plus avancé que le cancer de l'autre au moment du diagnostic. L'examen d'une douzaine d'articles confirme que l'association entre la privation sociale et la survie au cancer est due, au moins en partie, aux «différences en matière d'accès aux traitement optimaux» (Woods, Rachet \& Coleman, 2006, p.I6). La question cachée ou tue qu'il convient inequities in health Studies on social and economic determinants of population health, No. 2: WHO Collaborating Centre for Policy Research on Social Determinants of Health, University of Liverpool.

Young, I.M. (I990). Justice and the politics of difference. Princeton, NJ: Princeton University Press.

Young, I.M. (200I). Equality of whom? Social groups and judgments of injustice. Journal of Political Philosophy, 9(I), I-8.

de se poser est pourquoi: pourquoi les personnes qui sont défavorisées du point de vue socioéconomique bénéficient-elles moins souvent des traitements optimaux que leurs pairs plus favorisés dans les salles d'attente des centres de cancérologie?

Quelques-unes des explications sont relativement évidentes. Les dépenses personnelles engagées dans le cadre des soins contre le cancer peuvent être considérables. Les frais de transport, par exemple, peuvent grimper rapidement. Une étude qualitative réalisée auprès de femmes à faible revenu a révélé que certaines d'entre elles sautaient des séances de traitement parce qu'elles ne pouvaient pas se permettre les frais de transport jusqu'au centre de cancérologie ou ceux des antinauséeux (Gould, 2004). Divers programmes visent à compenser les coûts associés au cancer, mais les patients ne sont pas toujours au courant de leur existence, et des coûts restent à la charge des patients.

Les préoccupations entourant la capacité de vivre à la maison sans assistance durant le traitement sont aussi pertinentes. Dans une étude auprès de femmes diagnostiquées d'un cancer alors qu'elles avaient entre 70 et 89 ans, certaines femmes choisissaient de ne pas suivre les traitements recommandés (Sinding \& Wiernikowski, 2009). Irene expliquait sa décision de cette manière:

La qualité de vie sous chimio ne me disait rien qui vaille. Je vis seule et j'aime ça et je connais beaucoup de gens qui l'ont reçue et qui dépendaient de quiconque se trouvait à proximité ... Ma coiffeuse m'a parlé de son ami et de la façon dont ses enfants l'ont abandonné et que c'était rendu au point où il n'avait presque rien à manger (p. 626).

Quand Irene prenait des décisions sur le traitement, elle tenait compte du genre de soutien auquel elle pouvait s'attendre chez elle. Sa décision de renoncer à la chimiothérapie, qui est personnelle à un certain niveau, survient dans un contexte plus vaste: depuis quelques années, les deniers publics affectés aux soins à domicile (et l'éventail de ce qui est couvert) se sont amenuisés (Aronson \& Neysmith, 200I). Les travailleurs sociaux des centres de cancérologie aident les patients à cerner et à mobiliser des soutiens formels et informels mais, ici encore, les patients ne sont pas toujours aiguillés vers les soins de soutien, et les ressources communautaires ne sont pas toujours adéquates.

Une autre explication des disparités en matière de traitement anticancéreux tient à l'idée selon laquelle certains groupes de patients sont «passifs», qu'ils participent moins 
activement à leurs soins de santé ou qu'ils sont moins actifs dans la recherche d'information ou dans la demande de ressources de santé. Il s'agit là d'une théorie qui soulève la question: exigeons-nous que les patients agissent d'une manière particulière pour recevoir des soins de santé? Cela semble absurde, mais ce message est véhiculé de plus en plus fréquemment. Les dépliants des salles d'attente et certains sites Web encouragent les personnes atteintes de cancer à se procurer une copie de leur dossier et de leurs bilans de santé, de suivre leurs résultats d'examens, d'appeler pour faire le suivi de leurs rendez-vous et de savoir quels symptômes méritent de l'attention et de la part de qui. Le message adressé aux patients est qu'ils doivent «être actifs», qu'ils doivent «se prendre en mains » s'ils veulent recevoir les soins dont ils ont besoin.

C'est un problème pour les personnes atteintes de cancer et c'est également un problème pour ce qui est de l'équité. Notre équipe de recherche a interviewé des femmes atteintes d'un cancer du sein et se situant aux deux extrémités de l'échelle socio-économique, afin d'examiner attentivement le travail qu'elles faisaient relativement aux processus, au calendrier et à la qualité des soins. Nous avons découvert que certaines femmes semblaient concrétiser les promesses de «l'autonomisation des patients »; elles relataient des histoires dans lesquelles elles indiquaient avoir obtenu, par leurs propres actions, des soins plus nombreux, plus rapides et meilleurs. Cependant, lors de l'examen de ces histoires, nous avons dégagé des liens constants entre ce genre d'implication «réussie» et des capacités et ressources particulières: maîtrise de l'anglais oral, ressources matérielles, bonne connaissance du système de soins de santé et bonnes connaissances médicales, expérience et confiance dans la capacité à s'y retrouver au sein d'institutions et enfin, rôles associés aux postes de professionnels (Sinding, Miller, Hudak, Keller-Olaman, \& Sussman, 20I2). En somme, les patients plus favorisés ont plus de facilité à manipuler le système pour obtenir ce dont ils ont besoin. Ceci n'aurait guère d'importance si les professionnels de la santé étaient capables de fournir la coordination et le monitorage des soins que nous sommes en droit d'attendre de leur part. Mais lorsque les professionnels sont sollicités au maximum et n'accordent aucune attention particulière à la qualité des soins dispensés aux patients les plus marginalisés, des disparités peuvent survenir.

Les disparités se produisent également pour d'autres raisons. Dans une récente étude (Sinding et al., 2013), des travailleurs sociaux étaient interviewés à propos de leurs expériences de travail auprès de personnes ayant un double

\section{RÉFÉRENCES}

Aronson, J., \& Neysmith, S. (200I). Manufacturing social exclusion in the home care market. Canadian Public Policy, XXVII(2), I5I-I65.

Brophy, J., Keith, M., Watterson, A., Park, R., Gilbertson, M., Maticka-Tyndale, E., et al. (2OI2). Breast cancer risk in relation to occupations with exposure to carcinogens and endocrine diagnostic de maladie mentale grave et de cancer. Dans plusieurs situations, le diagnostic de maladie mentale-l'étiquetage dans le dossier - signifiait que les équipes de santé n'ont pas su reconnaître les symptômes qui se sont avérés être liés au cancer. Une travailleuse sociale mentionnait une patiente que son médecin de famille avait renoncé à soigner du fait de sa dépression et qu'il n'avait pas investigué ses symptômes physiques pourtant anciens (qui ont fait, plus tard, l'objet d'un diagnostic de cancer avancé). Une autre a décrit une situation dans laquelle des symptômes physiques particuliers ont été attribués au trouble de santé mentale (alors que le patient avait une tumeur cérébrale). Un patient souffrant de douleur persistante a été rejeté comme étant un toxicomane et n'a pas reçu le médicament dont il avait pourtant besoin, aux dires de la travailleuse sociale que nous avons interviewée.

Le «masquage du diagnostic» est le terme qui s'applique aux situations où le personnel soignant attribue les symptômes rapportés par un patient à son diagnostic de maladie mentale et fait donc abstraction de problèmes de santé importants ou les néglige. Et bien qu'il y ait des preuves de «masquage du diagnostic» en cancérologie, on sait aussi que les équipes de soins de santé travaillent activement avec certains patients pour qu'ils reçoivent leur traitement contre le cancer en consultant d'autres prestataires de soins du patient et en faisant tout pour apaiser les craintes du patient, souvent en faisant participer au processus une personne ayant la confiance du patient. Un article décrivait la façon dont une équipe d'oncologie accompagnait les personnes craignant particulièrement les rayonnements ( $\mathrm{du}$ fait de leurs circonstances de vie ou de certaines déficiences), les menait à travers la zone de traitement afin qu'elles se familiarisent avec l'espace et expriment leurs préoccupations, le tout de manière à ce que leur crainte soit atténuée et que le traitement puisse avoir lieu (Howard et al., 20I0).

Les prestataires de soins peuvent intervenir - ce qu'ils font d'ailleurs souvent — dans les processus favorisant l'apparition des disparités en matière de soins de santé. Ils trouvent souvent des moyens d'accueillir chaleureusement dans les milieux de soins toutes sortes d'individus atteints de cancer afin d'offrir des chances égales concernant l'accès aux soins et de remettre en question les pratiques d'exclusion par trop communes. Grâce au soutien des gestionnaires du système de santé et à des engagements explicites des institutions en faveur de l'équité, les infirmiers et infirmières en oncologie jouent un rôle vital en veillant à ce que les différences entre individus ne débouchent pas sur des disparités en cancérologie.

disruptors: A Canadian case-control study. Environmental Health, 11(I), 87 .

Gould, J. (2004). Lower-income women with breast cancer: Interacting with cancer treatment and income security systems. Canadian Woman Studies, 24(I), 3I-36. 
Howard, L.M., Barley, E.A., Davies, E., Rigg, A., Lempp, H., Rose, D., et al. (20IO). Cancer diagnosis in people with severe mental illness: Practical and ethical issues. Lancet Oncol, 11(8), 797-804.

Sinding, C., Miller, P., Hudak, P., Keller-Olaman, S., \& Sussman, J. (20I2). Of time and troubles: Patient involvement and the production of health care disparities. Health: An Interdisciplinary Journal for the Social Study of Health, Illness and Medicine 16(4), 400-4I7.

Sinding, C., Watt, L., Miller, P., Silliker, J., Lawson, L., Kislinsky, C., et al. (2013). Stigmas and silos: Social workers' accounts of care for people diagnosed with serious mental illness and cancer. Social
Work in Mental Health, 11(3), 288-309.

Sinding, C., \& Wiernikowski, J. (2009). Treatment decision making and its discontents. Social Work in Health Care, 48(6), 6I4-634.

Stanford, J. (2008). Economics for everyone: A short guide to the economics of capitalism. London: Pluto Press.

Steingraber, S., (20I4). Living Downstream. Retrieved from http:// steingraber.com/film

Woods, L., Rachet, B., \& Coleman, M. (2006). Origins of socioeconomic inequalities in cancer survival: A review. Annals of Oncology, 17(I), 5-I9.

\section{Disparités en matière de santé en oncologie: une perspective canadienne}

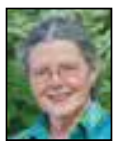

par Margaret Fitch, inf., Ph.D.

Experte, Résultats signalés par les patients et Survie Partenariat canadien contre le cancer

$\mathrm{L}$ e Partenariat canadien contre le cancer est avant tout un _ partenariat de spécialistes du cancer, d'organismes caritatifs, de gouvernements, de patients et de survivants, résolus à apporter des changements dans le domaine de la lutte contre le cancer. Le Partenariat a pour objectif principal de mettre en œuvre dans l'ensemble du Canada les meilleures connaissances disponibles sur la lutte contre le cancer.

Le Groupe d'action pour l'expérience globale du cancer avait pour mission de prendre la tête d'un effort visant à changer le point de mire des soins en cancérologie afin de mieux répondre aux besoins des patients et de leurs proches. Nous voulions optimiser et la qualité et l'accès, et par là même rehausser la qualité de vie des personnes touchées par le cancer et améliorer l'expérience globale du cancer parmi la population canadienne. Nous avons concentré notre attention sur l'implantation d'une approche centrée sur la personne au sein de la lutte contre le cancer. Cette approche exige que la fourniture de soins soit respectueuse des préférences, valeurs et besoins individuels et y soit sensible et il importe que ces valeurs guident l'ensemble des décisions cliniques. Cela signifie qu'il faut consciemment adopter la perspective de la personne quant à ce qui importe à ses yeux et à ce qui compte pour elle et travailler avec les personnes plutôt que d'agir sur elles ou pour elles.

Plusieurs rapports commandés par le Groupe d'action pour l'expérience globale du cancer abordent la question des disparités relativement au cancer. Chacun d'eux sera examiné ci-dessous.

I) Providing culturally competent supportive care for underserved populations [Fournir des soins de soutien culturellement adaptés aux populations mal desservies]

Cette recension de la littérature sur les meilleures pratiques en soins de soutien à l'intention des populations mal desservies a dégagé de nombreuses lacunes dans la prestation des soins de santé ainsi que les domaines nécessitant une étude plus poussée. Certaines interventions ont été cernées comme étant efficaces (intervenants pivots, approche communautaire, groupes de soutien des personnes atteintes d'un cancer vivant en région éloignée/à distance) et potentiellement efficaces (groupes de soutien face à face, groupes de soutien par le biais d'Internet, groupes de soutien et de counseling individuel par un pair, plateformes de télésanté, et enfin, équipes de sport/ activités récréatives/de compétition) dans la fourniture de soins adaptés sur le plan culturel. Les interventions sous-étudiées comprenaient les soins spirituels et palliatifs chez les populations mal desservies.

Le rapport soulignait les éléments essentiels des programmes adaptés à la culture: l'information est fournie de manière culturellement appropriée, on prête attention au niveau d'alphabétisation, on personnalise les messages en fonction des populations, on reconnaît les besoins spirituels, on implique des membres de la communauté, on utilise des intervenants pivots provenant de la population concernée et enfin, on implique des membres de la famille élargie.

Le rapport s'intéressait également aux services d'interprétation. Ces services ne sont toujours pas offerts dans beaucoup de centres de cancérologie. Jusqu'à présent, on ne dispose pas de preuves à l'appui d'un modèle particulier comme étant le plus efficace et les avantages/coûts des services sont essentiellement méconnus. Cependant, il est manifeste que les professionnels de la santé n'ont reçu aucune formation quant à l'utilisation d'interprètes et à la collaboration efficace avec eux.

2) Supportive care resources and services for non-Englishspeaking populations in Canada [Ressources et services en matière de soins de soutien pour les populations ne maîtrisant pas l'anglais au Canada]

Ce rapport décrivait l'expérience des populations ne maîtrisant pas l'anglais tandis qu'elles interagissaient avec les professionnels de la santé anglophones et la façon dont la gêne qu'elles ressentaient influençait leurs décisions sur la quête ou non d'information et/ou de soins ainsi que le moment choisi à ces fins. Beaucoup ne sont pas au courant des ressources qui existent et ne demandent pas ce qui est disponible pour les aider. Les services à l'intention des personnes ne maîtrisant pas l'anglais sont plutôt sporadiques et épars à travers le Canada, et il existe des obstacles financiers sur le plan de l'accès aux programmes. Il est manifeste que des besoins criants se font sentir en matière de services d'interprétation et de traduction efficaces, de programmation au niveau communautaire et de considération des remèdes traditionnels. 
3) Cancer care for ALL Canadians: improving access and minimizing disparities for vulnerable populations in Canada [Soins de cancérologie pour TOUS les Canadiens: améliorer l'accès et minimiser les disparités chez les populations vulnérables du Canada]

Ce rapport souligne qu'il est erroné de faire de quelconques suppositions quant aux services qui s'avéreront efficaces pour des populations vulnérables. Il encourage les planificateurs en soins de santé à impliquer les membres de la population dans leurs propres soins et dans la planification et la conception des services de santé. Il est essentiel d'aller dans la communauté afin de bien comprendre les populations et de leur fournir des services. "Même quand des preuves concluantes existent, les enjeux liés à la diversité culturelle, aux barrières linguistiques et à l'expérience des populations marginalisées sont rarement intégrés dans la planification des politiques et la pratique.» (Sarah Bowen, Winnipeg)

Dispenser des soins adaptés sur le plan culturel exige une compréhension de la culture (caractéristiques originales que nous possédons tous et qui nous distinguent à titre d'individus et qui signalent notre appartenance à un ou à plusieurs groupes) ainsi qu'un savoir-faire culturel (lequel met en jeu un ensemble de comportements, d'attitudes et de politiques qui permettent d'œuvrer efficacement dans des situations interculturelles; inclut la capacité à satisfaire avec respect et efficacité les besoins particuliers de populations diversifiées sur les plans culturel et linguistique; et enfin, garantit l'adaptation, la sensibilisation et la sécurité culturelles). Les principes des soins adaptés sur le plan culturel sont décrits dans le rapport.

Afin d'améliorer le système de lutte contre le cancer et de fournir des soins adaptés sur le plan culturel, il nous faut rehausser:

- Laccès (entrée dans le système, égalité au niveau des résultats, réceptivité, conception et mise en œuvre des programmes qui sont appropriés et pertinents)

- L'accès linguistique

- L'aide à la navigation des patients.

\section{4) Addressing disability in Canada [S'attaquer à l'incapacité au Canada]}

Dans l'Enquête postcensitaire sur la participation et les limitations d'activité (EPLA), Statistique Canada donne la définition suivante de l'incapacité: I) Une incapacité constitue une limitation d'activités ou une restriction rattachée à un état physique ou mental ou à un problème de santé; 2) la relation qui s'établit entre les structures et les fonctions corporelles, les activités de la vie quotidienne et la participation sociale tout en tenant compte du rôle des facteurs du milieu; 3) inclut les personnes qui éprouvent des limitations ou des obstacles relativement à la vue, louie, la mobilité, l'agilité, la douleur, la mémoire, l'apprentissage, le développement ou l'aspect émotionnel/psychologique.

Pour faire face aux incapacités et au cancer, il faut considérer deux populations:

- Les individus dont les incapacités existaient avant leur diagnostic de cancer (4,4 millions)

- Les individus dont les incapacités sont éprouvées du fait du cancer et/ou de son traitement (I,I million)
Ce rapport souligne les résultats d'entrevues et de groupes de discussion auprès des personnes ayant des incapacités liées à leur expérience de cancer. Les personnes atteintes d'une incapacité antérieurement à leur expérience de cancer signalaient: des retards dans la détection et le diagnostic, des expériences de traitement compliquées et une altération des choix de traitement. Ceux qui éprouvaient des incapacités à la suite du traitement de leur cancer décrivaient, entre autres: une paralysie faciale, une déficience auditive et l'endommagement d'organes après la radiothérapie; une réduction de la mobilité après l'amputation d'un ou de membre(s); des difficultés à manger et à boire, et enfin, des troubles de santé mentale et la fatigue. Les citations ci-dessous illustrent les types d'expériences vécus par ces personnes.

Expériences positives:

- J'ai dit «Bon, vous allez m’opérer. Si vous m’enlevez mes aides auditives, comment vais-je faire pour vous entendre?» Ils ont décidé de m'en laisser une. En fait, ils l'ont enlevée durant l'opération, mais ils me l'ont remise avant que je ne me réveille, ce qui veut dire que je pouvais entendre quand je me suis réveillé.

- Quand ils ont essayé de me parler à travers leurs masques, je leur ai dit « je n'arrive pas à comprendre ce que vous dites quand vous portez vos masques. [Je ne peux pas lire vos lèvres.]»Ils m'ont répondu qu'il leur était impossible de les enlever. Je leur ai donc dit «Dites-moi ce que vous allez faire avant que nous entrions là.»

Expériences difficiles:

- Je devenais vraiment faible et pâle, et je ressentais une énorme fatigue. J'en avais parlé aux médecins, mais ils ne cessaient de me dire que cétait ma sclérose en plaques qui empirait.

- On m'a dit que la douleur et tout ce que j'éprouvais étaient tout simplement liés à la trop grande quantité de temps que je passais dans mon fauteuil roulant ... mais je n'avais pas ces problèmes auparavant.

- Il était dur de lui expliquer le cancer et son traitement à cause de sa déficience intellectuelle. Comme il est plus âgé et plus grand maintenant, les gens ne se souviennent pas toujours du fait qu'il reste un enfant. [Parent]

- Quand on a diagnostiqué ma leucémie, mon oncologue n'avait jamais traité de personne atteintes de mon incapacité [la sclérose en plaques]... il a cherché partout pour trouver les principes devant guider le traitement... cela se réduisait à un processus d'essais et d'erreurs.

- Ma chirurgienne a choisi de réaliser une mastectomie radicale plutôt qu'une tumorectomie parce que j'utilise un insufflateur ... la radiothérapie pourrait avoir une incidence sur mes poumons.

- Je ne pouvais pas rester couché(e) sur le dos ou rester sans bouger pour la radiothérapie. Je fais des spasmes.

- Je suis sous dialyse et cétait fort difficile d'organiser la chimiothérapie en tenant compte de la dialyse.

- Lorsque j'ai démarré la chimiothérapie, j’ai dû dire à 35 intervenants la liste des médicaments que je prends et 
tous leurs dosages. Il m'a fallu parler de mes antécédents à chaque fois que je rencontrais un nouvel intervenant dans le cadre du traitement ... on m'a donné un médicament qui était censé réduire la nausée, mais il a interagi avec un de ceux que je prenais déjà ... c'était vraiment effroyable.

Les recommandations sur ce que l'on peut faire comprennent les suivantes:
- Réserver davantage de temps pour les rendez-vous et les traitements

- Rechercher l'aide des proches

- Faire en sorte que l'information soit plus accessible

- Adapter les milieux de traitement

- Coordonner les soins.

Les versions complètes de ces rapports en anglais sont disponibles à http://www.partnershipagainstcancer.ca

\section{Disparités en oncologie: Les défis et opportunités confrontant les États-Unis d'Amérique}

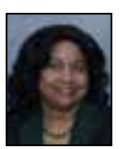

par Mary Magee Gullatte, Ph.D., inf., ANP-BC, AOCN, FAAN, Présidente de 2012 à 2014 et actuellement ancienne présidente, Oncology Nursing Society

Chaque année, l'American Cancer Society (ACS) publie des estimations des nouveaux cas de cancer et des décès par cancer parmi les différentes populations raciales et ethniques vivant aux États-Unis. Ces données résultent de la conglomération de diverses sources sur l'incidence du cancer, la mortalité, la survie et la prévalence du dépistage: National Center for Health Statistics, Centers for Disease Control, National Cancer Institute et le programme Surveillance, Epidemiology, and End Results (SEER). Les centres de cancérologie et les ministères de la Santé de tous les états américains soumettent leurs statistiques sur le cancer au service d'enregistrement du programme SEER. Les registres des tumeurs font le suivi des personnes atteintes de cancer et soumettent leurs données aux autorités de leur état qui les transmet ensuite au programme SEER.

En 20I4, on estimait que le nombre de nouveaux cas de cancer dépassait I,6 million aux États-Unis (Siegel, Ma, Zou, \& Jemal 20I4). Parmi ceux-ci, environ i76 620 survenaient chez des Afro-Américains; touchant davantage d'hommes que de femmes (DeSantis, Naishadham \& Ahmedin, 2013). La mortalité globale due au cancer se chiffrait, en 2013, à 580350 décès aux États-Unis (DeSantis et al., 2013) tandis que le nombre estimé de décès parmi les Afro-Américains s'élevait à 64880 (DeSantis et al., 2013).
Alors que le taux global de mortalité due au cancer décline actuellement et que le nombre de survivants du cancer dépasse I,3 million selon les estimations, on remarque des disparités fondées sur les données au niveau du nombre de nouveaux cas de cancer pour certains cancers et de la mortalité par cancer. Certains cancers touchent de manière disproportionnée les minorités et les populations mal desservies. Par exemple, l'incidence du cancer du sein chez les Afro-Américaines est plus faible que chez les Américaines de race blanche, et pourtant, la mortalité est plus élevée chez ces premières. Chez les AfroAméricains, on constate une disparité inexpliquée quant à l'incidence et à la mortalité du cancer de la prostate.

Les National Institutes of Health (NIH) des É.-U. définissent les disparités comme étant «des différences dans l'incidence, la prévalence, la mortalité, le fardeau des maladies et des autres effets néfastes qui existent parmi des groupes de population particuliers aux États-Unis » [traduction libre] (NIH, 20I4). La déclaration de l'initiative Healthy People 20Iо [Une population en santé 20I0], présente un ensemble d'objectifs en matière de santé que les États-Unis devront atteindre durant la première décennie de ce nouveau siècle. Le cancer est évoqué à maintes reprises à titre de domaine prioritaire où il convient d'élaborer des initiatives visant à améliorer l'état de santé et à éliminer les disparités en matière de santé $(\mathrm{NIH}$, 20I4).

Selon les statistiques sur le cancer de l'American Cancer Society (20I3), les Afro-Américains sont moins susceptibles de survivre au cancer que les blancs. Les taux de survie relative à

Tableau 1: Taux d'incidence du cancer selon la race et l'appartenance ethnique, États-Unis, 2005 à 2009

\begin{tabular}{|c|c|c|c|c|c|}
\hline Siège & Blancs & Afro-Américains & $\begin{array}{l}\text { Asio-Américains et insulaires } \\
\text { des îles du Pacifique }\end{array}$ & $\begin{array}{l}\text { Amérindiens* } \\
\text { Autochtones de l'Alaska }\end{array}$ & $\begin{array}{l}\text { Hispano-I } \\
\text { Latino-Américains }\end{array}$ \\
\hline Tous: H/F & $543,1 / 424$ & $619,7 / 397$ & $328 / 286$ & $423 / 360$ & 419/333 \\
\hline Cancer du sein - Femmes & 123,3 & 118,0 & 85,9 & 89,1 & 93,0 \\
\hline Poumons et bronches $\mathrm{H} / \mathrm{F}$ & $21,2 / 11,2$ & $23,3 / 12,1$ & $10,1 / 5,1$ & $29,0 / 16,6$ & $19,8 / 11,4$ \\
\hline \multicolumn{6}{|c|}{$\begin{array}{l}\text { * Il s'agit des taux par } 100000 \text { personnes avec ajustement de l'âge en fonction de la populatio } \\
\text { l'appartenance ethnique ne s'excluent pas mutuellement dans le cas d'une origine hispanique. }\end{array}$} \\
\hline
\end{tabular}


Tableau 2: Mortalité par cancer en fonction de la race et de l'appartenance ethnique, États-Unis, 2005 à 2009

\begin{tabular}{|l|l|l|l|l|l|}
\hline Siège & Blancs & Afro-Américains & $\begin{array}{l}\text { Asio-Américains et insulaires } \\
\text { des îles du Pacifique }\end{array}$ & $\begin{array}{l}\text { Amérindiens * } \\
\text { Autochtones de l'Alaska }\end{array}$ & $\begin{array}{l}\text { Hispano-I } \\
\text { Latino-Américains }\end{array}$ \\
\hline Tous: H /F & $217 / 151$ & $288 / 175$ & $133 / 93,2$ & $185 / 136$ & $146 / 101$ \\
\hline Cancer du sein - Femmes & 22,4 & 31,6 & 11,9 & 16,6 & 14,9 \\
\hline Poumons et bronches H/F & $65,3 / 40 / 8$ & $82,6 / 38,0$ & $35,9 / 18,5$ & $48,3 / 33,2$ & $30,8 / 14,1$ \\
\hline Prostate & 21,7 & 53,1 & 10,0 & 19,7 & 17,8 \\
\hline
\end{tabular}

* Il s'agit des taux par 100000 personnes avec ajustement de l'âge en fonction de la population américaine standard de 2000. La race et

l'appartenance ethnique ne s'excluent pas mutuellement dans le cas d'une origine hispanique.

Ca Cancer Journal for Clinicians (2013). Cancer Statistics, 2013, American Cancer Society, 62(1), 25.

cing ans sont plus bas pour presque tous les stades de cancer. Les tableaux I et 2 illustrent les données de l'ACS liées à l'incidence et à la mortalité selon la race et le sexe aux États-Unis entre 2005 et 2009 .

\section{Facteurs contribuant aux disparités en matière de santé}

On pense que plusieurs facteurs économiques et sociaux expliquent, en partie, les disparités en matière de santé constatées parmi les minorités raciales et ethniques et les groupes d'Américains mal desservis. Ils comprennent: (I) des iniquités dans l'accès aux soins de santé comme le dépistage, la détection précoce et le traitement [on dénombre 46 millions d'Américains qui sont sous-assurés ou encore non assurés]; (2) des iniquités au niveau de la qualité des soins à l'intérieur des établissements de soins et de l'accès aux essais cliniques; (3) la moindre susceptibilité d'être diagnostiqués à un stade précoce; et (4) la moindre susceptibilité de recevoir les soins oncologiques standards que les blancs pour les cancers du poumon, du sein et de la prostate et pour le cancer colorectal (Gullatte, Brawley, Kinney, Powe, \& Mooney, 2010; Underwood \& Powell, 2006).

\section{Réduire les disparités quant à l'incidence et à la mortalité du cancer}

Les infirmiers et infirmières en oncologie sont des membres essentiels de l'équipe interprofessionnelle. Ils sont bien placés pour faire une différence relativement à la réduction et l'élimination des disparités en matière de santé et pour améliorer les résultats des soins oncologiques. Le CDC et d'autres services de santé publique, les prestataires de soins et les communautés de l'ensemble des groupes raciaux et ethniques doivent œuvrer au sein d'un effort national pour:

I. améliorer la détection précoce du cancer grâce aux mammographies, tests Pap et dépistage du cancer colorectal, tous réalisés de façon routinière;

2. mettre en œuvre des interventions communautaires fondées sur des données probantes afin d'accroître le dépistage et de modifier les comportements à risque;

3. concevoir des projets de recherche qui encourageront les groupes minoritaires à participer à des essais cliniques dans le cadre de la prévention du cancer afin de cerner des différences significatives parmi les groupes minoritaires et ethniques;
4. réaliser des recherches qui éclaireront les décisions sur les interventions en vue de réduire les disparités liées au cancer et de rehausser l'état de santé (on constate actuellement un manque de données sur les interventions mises à la disposition des gens quel que soit leur statut socio-économique ou leur comportement et abordant aussi l'environnement social); et,

5. utiliser un éventail de médias et de véhicules pour faire passer l'information sur le cancer à des populations diverses et dans toute une gamme de contextes.

Signaler les retards en matière de détection précoce pour le cancer du sein chez les Afro-Américaines

Les retards dans la détection du cancer du sein ont été signalés comme facteur important dans les cancers de stade avancé (Bibb, 200I; Burgess et al., I998 \& 200I). Une étude multi-méthodes de Gullatte et al. (2010) a permis de mettre au jour une différence statistiquement significative concernant les retards de recherche de soins médicaux pour les changements auto-détectés au niveau des seins et le stade du cancer du sein. Létude examinait les croyances religieuses et spirituelles des Afro-Américaines et la probabilité de tarder à chercher des soins médicaux. On constatait une différence statistiquement significative concernant la recherche de soins médicaux chez les femmes qui parlaient uniquement à Dieu du changement survenu au niveau de leurs seins. La durée de ce retard parmi les participantes à l'étude s'étendait de quelques semaines à I8 mois. L'étude a en outre analysé les croyances fatalistes sur le cancer parmi les Afro-Américaines ayant survécu au cancer du sein et subissant leur première année de traitement. Quoique les femmes soient très religieuses, on ne remarquait aucune différence statistiquement significative en lien avec des croyances fatalistes sur le cancer.

Les participantes à l'étude étaient un échantillon de commodité de 129 femmes, ayant entre 30 et 84 ans, qui indiquaient avoir détecté elles-mêmes un symptôme au niveau de leurs seins avant qu'un diagnostic de cancer du sein soit posé et dans les I2 mois précédant la conduite de l'étude. Celle-ci représentait une avancée capitale dans les efforts déployés pour dégager la profondeur à laquelle les croyances religieuses et spirituelles de certaines Afro-Américaines pouvaient exercer une incidence négative sur la mortalité par cancer en fonction du retard dans la quête de soins médicaux. Il n'existe à l'heure actuelle aucune mesure rigoureuse cohérente de l'influence des croyances 
religieuses et spirituelles sur le temps mis à rechercher des soins médicaux lors de la perception de changements au niveau des seins par des Afro-Américaines. On n'a trouvé aucune étude qui mesurait spécifiquement l'influence des croyances religieuses, spirituelles et reliées au fatalisme sur le temps mis à rechercher des soins médicaux pour des symptômes aux seins auto-détectés parmi cette population. La plupart des études de recherche indiquaient que les croyances religieuses, spirituelles ou reliées au fatalisme face au cancer constituaient des obstacles à l'intention des femmes à rechercher des soins médicaux; toutefois, les participantes ont fondé leurs réponses sur un scénario et non sur une expérience vécue. Le retard au chapitre du diagnostic représente un problème chez les Afro-Américaines. Cette conclusion rejoint les résultats d'autres études dans lesquelles le retard de traitement depuis la découverte d'un symptôme se traduit par un stade de cancer plus avancé lors du diagnostic (Richards, Westcombe, Love, Littlejohns \& Ramirez, I999; Facione \& Giancarlo, I998).

Il importe donc que les prestataires de soins aient conscience de ces données et saisissent toutes les occasions d'intervenir auprès des Afro-Américaines en termes de risque de cancer du sein, de dépistage, de reconnaissance des symptômes et de détection précoce en la matière. Des interventions axées sur la foi ont permis de véhiculer des messages sur la santé auprès d'Afro-Américaines. Une autre approche pourrait être d'établir des partenariats avec des ecclésiastiques afin de les instruire sur les problèmes éprouvés par les AfroAméricaines qui choisissent exclusivement l'intervention religieuse ou retarde le traitement conventionnel en attendant l'intervention religieuse. Beaucoup de ces femmes s'étaient bel et bien entretenues avec leurs ecclésiastiques et bénéficiaient de prières, mais il était possible que personne ne leur suggérait de se prévaloir également d'un traitement médical. Les ecclésiastiques peuvent jouer un rôle déterminant en encourageant les femmes qui les consultent à des fins religieuses à se rechercher également une intervention médicale. Les ecclésiastiques pourraient jouer un rôle capital en aidant les femmes à réaliser que les soins médicaux sont en harmonie avec leurs croyances religieuses et spirituelles en matière de guérison.

Les femmes qui avaient une histoire familiale de cancer du sein étaient plus susceptibles de rechercher des soins médicaux que les femmes qui indiquaient ne pas avoir d'antécédents familiaux. Ceci explique la nécessité éventuelle d'accroître l'éducation des femmes relativement aux symptômes $\mathrm{du}$ cancer $\mathrm{du}$ sein et d'assurer leur observation des lignes directrices en matière de dépistage de ce cancer.

Il faudra réaliser des études afin de comprendre l'influence de l'attitude religieuse, de la spiritualité et du fatalisme face au cancer au moment de la découverte de symptômes. Cette étude est limitée par le fait qu'on a mesuré les construits après que les patientes se soient mises en quête d'un diagnostic et d'un traitement médicaux; on ne sait pas si les scores relatifs aux mesures avaient changé depuis la détection initiale de symptômes. Une telle étude serait plutôt difficile à réaliser. Peut-être qu'en lançant un appel aux femmes dans le cadre d'un programme de santé axé sur la foi, on pourrait recruter des femmes ayant remarqué divers changements au niveau de leurs seins ou, en choisissant une option moins parfaite mais plus pratique, on pourrait recruter les femmes dès qu'elles sont passées à l'action en réaction à un changement observé, mais avant la mammographie diagnostique.

Il serait bon de faire une étude plus poussée des facteurs qui sont associés avec de plus brefs délais dans la recherche de soins médicaux. Le rôle du soutien social et déterminer si le fait d'encourager les femmes de parler à quelqu'un d'autre des changements affectant leurs seins aboutirait à des retards moins importants pourraient constituer une nouvelle voie d'investigation.

Il conviendrait en outre de mener des études qualitatives plus poussées afin de comprendre les expériences des femmes ayant de fortes croyances religieuses et spirituelles mais qui ne tardent pas à chercher des soins médicaux, d'une part, et celles qui présentent le même profil et tardent à le faire. Cela pourrait fournir des indications utiles sur la façon dont les croyances religieuses contrecarrent les actions de certaines mais pas d'autres. Il est nécessaire de concevoir de nouvelles stratégies d'éducation et de soutien aux Afro-Américaines afin qu'elles se servent de leurs croyances religieuses et spirituelles pour compléter l'intervention médicale plutôt qu'en remplacement de cette dernière. Par exemple, «s'en remettre aux mains de Dieu» ne doit pas nécessairement signifier «à la place» des soins médicaux. Il faut qu'elles comprennent que de «remettre cela aux mains de Dieu», «cela» se rapportant aux symptômes physiques, pourrait vouloir dire rechercher des soins médicaux le plus tôt possible et non le contraire. Dans l'optique d'une intervention communautaire, la collaboration avec des ecclésiastiques des lieux de culte afro-américains en vue d'instruire les femmes sur la santé et la guérison, pourrait mener à une réduction du retard à se faire soigner dans le cas de changements aux seins auto-détectés.

\section{Stratégies efficaces visant à réduire les disparités et à améliorer les résultats}

Les stratégies dont les infirmiers et infirmières en oncologie et les membres de l'équipe interprofessionnelle peuvent se servir pour résoudre les obstacles courants en matière de soins aux personnes atteintes de cancer et pour améliorer les résultats comprennent les suivantes: (I) étendre l'accès aux soins oncologiques optimaux et aux essais cliniques afin de veiller à ce que les groupes minoritaires reçoivent les mêmes soins et le même accès à la technologie de pointe dont bénéficient les patients des grands centres de cancérologie; (2) aborder la peur du cancer, le coût toujours plus élevé des soins et le manque d'orientation vers des prestataires de soins; (3) offrir des services de dépistage à toutes les personnes qui en ont besoin; et (4) recommander l'emploi accru des services de prévention (Centers for Disease Control). Les National Institutes of Health des États-Unis ont une division consacrée à la santé chez les minorités et aux disparités en matière de santé dont la mission est de mener des recherches scientifiques en vue d'améliorer l'état de santé des membres des minorités et d'éliminer les disparités en matière de santé. On espère que la Patient Protection and Affordable Care Act [Loi sur la protection des patients et les soins abordables], adoptée le 23 mars 20Io, aidera à atténuer la distribution inégale du fardeau du cancer et d'autres maladies parmi les populations pauvres et mal desservies des É.-U. quand davantage d'Américains auront accès aux soins par le biais d'une couverture d'assurance-maladie abordable. 


\section{RÉFÉRENCES}

American Cancer Society (2013). Cancer facts Q figures 2013. Retrieved from http://www.cancer.org/acs/groups/content/@ epidemiologysurveilance/documents/document/acspc-036845.pdf

American Cancer Society. (2013). Cancer facts and figures for African Americans 2013-2014. Atlanta: American Cancer Society.

American Cancer Society (20I3). Cancer Statistics, 20I3. CA Cancer Journal for Clinicians, 62(I), II-30.

Bibb, S.C. (200I). The relationship between access and stage at diagnosis of breast cancer in African American and Caucasian women. Oncology Nursing Forum, 28(4), 7II-7I9.

Burgess, C.C., Hunter, M., \& Ramirez, A. (200I). A qualitative study of delay among reporting symptoms of breast cancer. The British Journal of General Practice, 77(8), 967-971.

Burgess, C.C., Ramirez, A., Richards, M., \& Love, S. (I998). Who and what influences delayed presentation in breast cancer? British Journal of Cancer, 77, I343-1348.

Centers for Disease Control (20I3). Health disparities. Retrieved from http:// www.cdc.gov/cancer/healthdisparities/basic_info/disparities.htm

Centers for Disease Control (20I4) Preventing disparities in cancer care. Retrieved from http://www.cdc.gov/nccdphp/

DeSantis, C., Naishadham, D., \& Jemal, A. (2013). Cancer statistics for African Americans, 20I3. CA Cancer Journal for Clinicians, 13(3) I5I-I66.
Facione, N.C., \& Giancarlo, C.A. (I998). Narratives of breast symptom discovery and cancer diagnosis: Psychologic risk for advanced cancer at diagnosis. Cancer Nursing, 21, 430-440.

Gullatte, M., Brawley, O., Kinney, A., Powe, B., \& Mooney, K. (2010). Influence of religiosity, spirituality, and cancer fatalism beliefs on delay in breast cancer detection in African American women. Journal of Religion and Health, 49(1), 62-72.

National Institute of Health (20I4). Health people 2020. Retrieved from http://www.healthypeople.gov

Phillips, J.M., Cohen, M.Z., \& Moses, G. (I999). Breast cancer screening and African American women: Fear, fatalism, and silence. Oncology Nursing Forum, 26, 56I-57I.

Richards, M., Westcombe, A., Love, S., Littlejohns, P., \& Ramirez, A. (I999). Influence of delay on survival in patients with breast cancer: A systematic review of literature. The Lancet, 353, III9-II26.

Siegel, R., Ma, J., Zou, Z., \& Jemal, A. (20I4). Cancer statistics, 2014. CA Cancer Journal for Clinicians, 64(1) 9-29.

Underwood, S., \& Powell, R. (2006). Religion and spirituality: Influence on health/risk behavior and cancer screening behavior of African Americans. Association of Black Nursing Faculty Journal, 17, 20-3I.

\section{Disparités en matière de santé en oncologie: perspectives internationales}

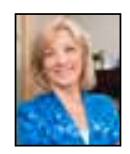

Greta G. Cummings, inf., Ph.D., FCAHS

Présidente de $20 \mathrm{IO}$ à $20 \mathrm{I} 4$ et présentement présidente sortante, International Society of Nurses in Cancer Care (ISNCC)

$\mathrm{N}$ ous vivons dans un monde où les disparités s'accroissent régulièrement. Ainsi, plus de $\mathrm{I}, 3$ milliard de personnes n'ont pas accès à de l'eau potable, 3 milliards ne disposent pas d'installations sanitaires adéquates, 2 milliards n'ont pas accès à l'électricité, et enfin, 3 milliards vivent avec moins de $2 \$$ par jour (World Development Indicators, 2003). Aussi récemment qu'en l'an 2000, un milliard de personnes ne savaient ni lire ni signer leur nom (INTCR, 20I3). L'incidence de ces disparités est accablante. Environ II millions d'enfants meurent chaque année pour cause de pauvreté (UNICEF, 2006), et un autre million d'enfants par manque d'eau potable et d'installations sanitaires adéquates (UN, 2008).

Les disparités découlent d'une multitude de facteurs, notamment le statut socio-économique, l'appartenance ethnique, les ressources des pays et les services de diagnostic, de traitement et de palliation dans le cadre du cancer. A titre d'exemple, en Angleterre, les gens qui habitent des quartiers défavorisés recevaient 70\% moins de soins de santé par rapport à leurs besoins que les gens des quartiers opulents (WHO, 2008). Des disparités ethniques ont été constatés lorsque 2946 patients de race blanche et 367 patients de race noire, âgés de 65 ans et plus, ont été diagnostiqués d'un cancer de l'œsophage d'extension locorégionale; les patients âgés de race noire étaient moins susceptibles de consulter un chirurgien, un radio-oncologue ou un oncologue médical à la suite de leur diagnostic de cancer de l'œsophage (Steyerberg 2007).
Ashford et Collymore (2005) donnent un exemple de disparités associées aux ressources des nations. La plupart des cas de cancer du col utérin peuvent être prévenus ou traités efficacement et pourtant, 274000 femmes meurent annuellement de cette maladie; parmi ces décès, 24I 000 se produisent dans des pays à revenu allant de faible à moyen. Les disparités en matière de traitement du cancer se reflètent dans la prise en charge de la douleur. Quelque $80 \%$ de la population mondiale, dont $>5$ millions de patients atteint d'un cancer terminal, n'ont pas accès à des traitements contre la douleur. Dans >I50 pays, la morphine n'est tout simplement pas disponible (Lamas \& Rosenbaum, 20I2). Les pays à revenu faible et à revenu moyen possèdent moins de $5 \%$ des ressources mondiales visant à lutter contre le cancer, bien que 70\% des décès par cancer y surviennent (WHO-Globocan, 20I2). Plus de $80 \%$ de leurs patients atteints de cancer sont déjà au-delà de toute guérison possible au moment du diagnostic (UICC, 20I3).

\section{L'International Society of Nurses in Cancer Care: gestion des disparités en matière de cancer}

Lapproche retenue par l'International Society of Nurses in Cancer Care (ISNCC) pour gérer les disparités en matière de cancer comprennent plusieurs initiatives stratégiques: défendre les intérêts des patients et influencer les politiques, souligner et clarifier le rôle et la contribution des infirmiers et infirmières en oncologie sur le plan de la lutte contre le cancer et des résultats, partager des ressources afin de former le personnel infirmier au renforcement des capacités, enseigner au personnel infirmier à défendre des causes, encourager des projets de recherche infirmière en soins oncologiques afin de développer le corpus des données probantes et d’appuyer l'utilisation de ces dernières. 
L'ISNCC prend des mesures en établissant des partenariats avec d'autres organismes du domaine du cancer d'envergure internationale et transnationale, puisque nous croyons que les ressources, la passion et l'influence ont davantage d'impact quand nous travaillons tous ensemble. Les partenaires internationaux de l'ISNCC comprennent l'International PsychoOncology Society (IPOS) - en vue de reconnaître et rehausser les rôles infirmiers et leur efficacité en psycho-oncologie; l'International Council of Nurses (ICN) - en vue d'influencer les politiques liées au cancer; l'Union for International Cancer Control/Union internationale contre le cancer (UICC) - pour l'action politique et enfin, la Multinational Association for Supportive Care in Cancer (MASCC) - en vue de reconnaître et rehausser les rôles infirmiers au sein des équipes multidisciplinaires en oncologie. Nous avons également des partenaires dans diverses régions du monde: European Oncology Nursing Society (EONS), Oncology Nursing Society (ONS), Asian Oncology Nursing Society (AONS), ainsi que des fondations et de grandes sociétés.

Un exemple de programme de partenariat avec l'EONS et la Bristol Myers Squibb Foundation s'intitule Gérer les disparités liées au cancer en Europe de l'Est. Parmi les projets présentés en $20 \mathrm{I} 3$ à la séance Bridging Cancer Care, à Amsterdam, PaysBas, on notait les suivants:

- Projet Espoir: Améliorer la détection précoce et les diagnostics de cancers pédiatriques dans cinq régions de la Pologne

- Renforcement des capacités du personnel infirmier de Roumanie en matière de soins en oncologie

- Encourager les Roms de Hongrie à s'impliquer dans la prévention et la détection précoce du cancer

- La formation en collaboration au service de l'amélioration de la sécurité et de la qualité des soins aux patients atteints de cancer en Russie

\section{RÉFÉRENCES}

Ashford, L. \& Collymore, Y. (2005). Preventing cervical cancer worldwide. Population Reference Bureau. http://www.prb.org/ pdfo5/preventcervcancer-brief_eng.pdf

Cummings, G.G. (20I2). Your leadership style-How are you working to achieve a preferred future? Journal of Clinical Nursing 2I, 3325-3327.

International Network for Cancer Treatment and Research (2013). Cancer in Developing Countries-Cancer-A Neglected Health Problem in Developing Countries. http://www.inctr.org/about-inctr/ cancer-in-developing-countries/

Krishnasamy, M. (2013). In a world of growing inequity-What now? International Cancer Nursing News (ICNN), 25(2), 4-6.

Lamas, D., \& Rosenbaum, L. (20I2). Painful inequities-Palliative care in developing countries. New England Journal of Medicine, 366, I99-20I.

Steyerberg, E.W., et al. (2007). Racial differences in surgical

\section{RÉFÉRENCE}

* Canadian Association of Nurses in Oncology/Association canadienne des infirmières en oncologie (ACIO/CANO) (20I3). CANO/ACIO strategic plan. Vancouver, BC: Author.
L'ISNCC renforce également les capacités en soins infirmiers en oncologie un peu partout dans le monde en concevant des programmes de formation des formateurs à l'intention du personnel infirmier dans le dépistage du cancer du col de l'utérus (avec l'UICC), la chimiothérapie par voie orale (avec Novartis et GSK) ainsi que des programmes de désaccoutumance au tabac (avec BMS Foundation, et les Drs Bialous et Sarna). Nous nous efforçons également de rejoindre le plus nombre possible d'infirmiers et infirmières en oncologie partout dans le monde puisque l'adhésion donne accès à l'information. En devenant membres, les infirmiers et infirmières peuvent acquérir des connaissances, accéder à des ressources et saisir diverses occasions d'influencer le cours des choses. Ceci exige des infirmières et infirmiers qu'ils fassent preuve de leadership (manifestent leur pouvoir) dans leur système de santé pour œuvrer en toute efficacité. Quelques-unes des approches de l'ISNCC consistent à aider le personnel infirmier à développer des associations professionnelles en soins infirmiers en oncologie dans leur localité ou pays, à influencer les politiques axées sur la lutte contre le cancer, à concevoir et présenter des programmes de formation des formateurs en vue de développer les capacités dans les soins infirmiers en oncologie et à fournir au personnel infirmier des occasions de développer des compétences en leadership. Le leadership signifie être capable de voir le présent tel qu'il est réellement et l'avenir tel qu'il pourrait être, puis de passer à l'action afin de combler l'écart [traduction libre] (Cummings, 20I2). Après avoir écouté les conseils de Mei Krishnasamy, conférencière Robert Tiffany lors de l'International Cancer Care Nursing Conference de 2012, voici quelquesunes des choses qui peuvent être faites afin de lutter contre les disparités: manifester de la vigilance face aux iniquités, œuvrer aux côtés de collègues pour mettre l'iniquité à l'avant-plan de votre discours sur les soins et pour mettre l'iniquité à l'avantplan du discours de votre équipe/unité/organisme.

evaluation, treatment and outcome of locoregional esophageal cancer: A population based analysis of elderly patients. Journal of Clinical Oncology, 25, 2389-96.

Union for International Cancer Control. (2013). World Cancer Declaration 2013. http://www.uicc.org/world-cancer-declaration

United Nations Development Programme (UNDP) (20I2). United Nations Human Development Report: Beyond Scarcity: Power, Poverty and the Global Water Crisis. UNDP. New York.

The World Bank (2003). World development indicators. http://wwwwds.worldbank.org

World Health Organization. (2008) Social determinants of health. http://www.who.int/social_determinants/en/

World Health Organization-Globocan (2012). Glococan 2012: Estimated Cancer Incidence, Mortality, and Prevalence Worldwide in 2012. http://globocan.iarc.fr/Pages/fact_sheets_cancer.aspx 